\title{
Oral administration of the milk casein-derived tripeptide Val-Pro-Pro attenuates high-fat diet-induced adipose tissue inflammation in mice
}

\author{
Kotaro Aihara ${ }^{1,2}$, Mizuko Osaka ${ }^{1}$ and Masayuki Yoshida ${ }^{1 *}$ \\ ${ }^{1}$ Department of Life Science and Bioethics, Graduate School of Medical and Dental Sciences, Tokyo Medical and \\ Dental University, MED Tower S954, 1-5-45 Yushima, Bunkyo-ku, Tokyo 113-8519, Japan \\ ${ }^{2}$ RED Center, Calpis Company Limited, 5-11-10 Fuchinobe, Chuo-ku, Sagamihara-shi, Kanagawa 252-0206, Japan \\ (Submitted 30 October 2013 - Final revision received 29 January 2014 - Accepted 26 February 2014 - First published online 28 May 2014)
}

\section{Abstract}

Inflammation of adipose tissue triggers the metabolic syndrome, atherosclerosis and CHD. In the present study, we investigated whether the milk casein-derived tripeptide valine-proline-proline (VPP) has an anti-inflammatory effect on the adipose tissue of high-fat diet (HFD)fed mice. Male C57BL/6J mice (7 weeks of age) were fed ad libitum with either a HFD and plain tap water (HFD group) or a HFD and water containing $0.3 \mathrm{mg} \mathrm{VPP} / \mathrm{ml}$ (HFD+VPP group) for 10 weeks. The results showed that the expression level of CD18 in the peripheral blood monocytes of the HFD+VPP group was significantly decreased compared with the level observed in those of the HFD group. Activated monocytes and pro-inflammatory macrophages were accumulated in the stromal vascular fractions of the adipose tissue from HFD-fed mice, which were significantly decreased in those supplemented with VPP. The formation of crown-like structures rich in pro-inflammatory macrophages was also significantly reduced in the adipose tissue of mice administered with VPP. Real-time PCR analysis revealed that the expression of monocyte chemoattractant protein- 1 and that of the pro-inflammatory cytokine $I L-6$ in adipose tissue tend to be lower in the HFD+VPP group than in the HFD group. These observations indicate that oral administration of VPP exerts an anti-inflammatory effect on the adipose tissue of HFD-fed mice, which may eventually lead to the primary prevention of chronic inflammation-related diseases.

Key words: Casein-derived tripeptides: Inflammation: High-fat diet: Adipose tissue: Macrophages

Lifestyle-related metabolic disorders such as hypertension, diabetes and dyslipidaemia play a crucial role in the development of atherosclerosis and CVD. Low-grade, but chronic inflammation is considered to be involved in the development of metabolic disorders. Among these, inflammation of adipose tissue is associated with obesity and directly promotes systemic low-grade inflammation and metabolic dysfunction ${ }^{(1-3)}$.

It has been reported that two naturally existing milk caseinderived bioactive tripeptides, isoleucine-proline-proline (IPP) and valine-proline-proline (VPP), produced during lactic acid bacteria fermentation or in casein hydrolysates, inhibit angiotensin-converting enzyme activity ${ }^{(4,5)}$, and foods containing these peptides reduce blood pressure in several clinical trials $^{(6,7)}$. VPP and IPP have also been shown to induce the production of vasodilative substances including $\mathrm{NO}$ in human umbilical vein endothelial cells and isolated rat arterial vessels ${ }^{(8)}$. Several recent clinical trials have shown that intake of casein hydrolysate containing VPP and IPP for 1 week improved vascular endothelial dysfunction without affecting systemic blood pressure in subjects with untreated stage-I hypertension ${ }^{(9)}$. Moreover, an 8-week intervention using the same regimen has been shown to decrease central systolic blood pressure and arterial stiffness (brachial-ankle pulse wave velocity and carotid arterial compliance $)^{(10,11)}$ Animal studies have revealed that in male $a p o E^{-/-}$mice, continuous intake of VPP, IPP or fermented milk, or casein hydrolysate containing these peptides, leads to a significant decrease in the development of atherosclerosis (intima to media thickness in the aortic arch) ${ }^{(12)}$. In vitro studies have shown that VPP and IPP attenuated phorbol 12-myristate 13-acetate (PMA)-stimulated adhesion of monocytic cells (THP-1) to activated human umbilical vein endothelial cells via suppression of the PMA-induced up-regulation of $\beta 1$ and $\beta 2$ integrin activation ${ }^{(13)}$. The mechanism of action may involve the inhibition of c-Jun N-terminal kinase phosphorylation in THP-1. These data suggest a distinct

\footnotetext{
Abbreviations: CCR2, CC chemokine receptor 2; FITC, fluorescein isothiocyanate; HFD, high-fat diet; ICAM-1, intercellular adhesion molecule-1; IPP, isoleucine-proline-proline; MCP-1, monocyte chemoattractant protein-1; NC, normal chow; PE, phycoerythrin; PMA, phorbol 12-myristate 13-acetate; SVF, stromal vascular fraction; VPP, valine-proline-proline.
} 
anti-inflammatory effect of VPP on vascular inflammation and atherosclerosis.

In the present study, we focus on the role of the VPP peptide in modulating obesity-related chronic inflammation under high-fat diet (HFD) conditions in vivo. We demonstrate that oral administration of VPP exerts an anti-inflammatory effect on the adipose tissue of HFD-fed mice via the inhibition of the accumulation of pro-inflammatory macrophages in the stromal vascular fraction (SVF).

\section{Materials and methods}

\section{Animals}

Male C57BL/6J mice (7 weeks of age; day 0) were obtained from Oriental Yeast Company Limited. They were fed with normal chow (NC; CLEA Japan, Inc.) or a HFD (20\% beef tallow and $1.25 \%$ cholesterol; CLEA Japan, Inc.). Mice fed with $\mathrm{NC}$ were given plain tap water (NC group) and those fed the HFD were given plain tap water (HFD group) or water containing $0.3 \mathrm{mg} \mathrm{VPP} / \mathrm{ml}$ (HFD+VPP group) for 10 weeks. VPP was provided by Calpis Company Limited.

Both food and water were provided ad libitum throughout the course of the experiments. Mice were euthanised under anaesthesia induced by an intraperitoneal injection of sodium pentobarbital $(50 \mathrm{mg} / \mathrm{kg})$. Blood samples were collected from the tail vein to measure blood glucose, insulin and lipid concentrations. Blood samples, epididymal adipose tissue and the liver were isolated and immediately frozen in liquid $\mathrm{N}_{2}$, and then stored at $-80^{\circ} \mathrm{C}$ until analysis

All animal experiments were approved by the Ethical Committee for Animal Experimentation of Tokyo Medical and Dental University, Tokyo and conducted according to the institutional guidelines.

\section{Flow cytometric analysis of circulating leucocytes}

Leucocytes were prepared by haemolysing whole blood. The cells were incubated with anti-mouse antibodies (fluorescein isothiocyanate (FITC)-CD11b, phycoerythrin (PE)-CD11c, FITC-CD29, PE-CD18, FITC-CD49d, FITC-F4/80 (BioLegend, Inc.); Alexa Flour 647-CD204 (AbD Serotec)) for $45 \mathrm{~min}$ on ice. After washing, fluorescence intensity was measured from cell fractions containing 5000 cells using a FACSCalibur (BD Biosciences), and data were analysed with FlowJo software (Tree Star, Inc.). When fluorescence activity was detected, data were obtained from monocytes using gating definitions, as reported previously ${ }^{(14-16)}$.

\section{Flow cytometric analysis of cell populations in the stromal vascular fractions of adipose tissue}

The SVF of each mouse was prepared as described previously $^{(16)}$. Briefly, the SVF was isolated from the adipose tissue of mice in the control, HFD and HFD+VPP groups after a test period of 10 weeks. Adipose tissue was excised, minced and incubated in PBS with heparin $(38.5 \mu \mathrm{g} / \mathrm{ml}$; $5 \mathrm{U} / \mathrm{ml}$ ) for $30 \mathrm{~s}$ to remove circulating blood cells, then the suspension was centrifuged at $1000 \mathrm{~g}$ for $8 \mathrm{~min}$ and the collected adipose tissue was incubated with type 2 collagenase

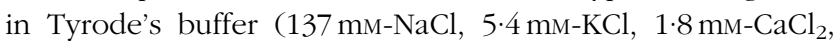


cose). The digested adipose tissue solution was centrifuged at $1000 \mathrm{~g}$ for $8 \mathrm{~min}$, and pellets containing the SVF were resuspended in PBS followed by filtration through a $70 \mu \mathrm{m}$ cell strainer (BD Biosciences). Isolated cells were incubated with anti-mouse antibodies (FITC-CD11b, PE-CD11c, FITC-F4/80 (BioLegend, Inc.); Alexa Flour 647-CD204 (AbD Serotec)) for $45 \mathrm{~min}$ on ice, followed by washing. Fluorescence intensity was measured from 5000 cell fractions using a FACSCalibur (BD Biosciences), and data were analysed with FlowJo software (Tree Star, Inc.). When fluorescence activity was detected, data were obtained from monocyte/macrophage subsets using gating definitions, as reported previously ${ }^{(16,17)}$. Cell populations in the SVF consisted of M1 macrophages $\left(\mathrm{F} 4 / 80^{+} / \mathrm{CD}_{11 \mathrm{c}^{+}}\right.$cells), M2 macrophages $\left(\mathrm{F} 4 / 80^{+} / \mathrm{CD} 204^{+}\right.$ cells) and activated monocytes $\left(\mathrm{CD} 11 \mathrm{~b}^{+} / \mathrm{CD} 11 \mathrm{c}^{+}\right.$cells), as reported previously ${ }^{(18-24)}$.

\section{Histological analysis of visceral adipose tissue}

At the end of each experiment, epididymal adipose tissue of mice were dissected and weighed. A portion of the tissue was fixed with $10 \%$ formalin in neutral buffer solution, and the fixed epididymal fat tissue was dehydrated through a graded ethanol series, embedded in paraffin, cut into $5 \mu \mathrm{m} \mathrm{sec-}$ tions, and stained with haematoxylin and eosin. For immunohistochemical analysis, the $5 \mu \mathrm{m}$ sections were deparaffinised and rehydrated, treated with $1 \%$ of $\mathrm{H}_{2} \mathrm{O}_{2}$ in methanol, and subjected to antigen retrieval in a high-pressure steamer, followed by blocking in skimmed milk. Subsequently, the sections were incubated with anti-mouse F4/80 antibodies (BioLegend, Inc.) overnight at $4^{\circ} \mathrm{C}$. After washing, they were probed with Histofine ${ }^{\circledR}$ Simple Stain Mouse MAX PO (Rat) (Nichirei Corporation) and then stained with the peroxidase substrate diaminobenzidine (Dako Denmark A/S), followed by counterstaining with haematoxylin and examination under a light microscope (Olympus IX71; Olympus Corporation) equipped with a SPOT RT Color-2000 digital camera (Diagnostic Instruments, Inc.). Crown-like structures were quantified in five random fields in each group of animals.

\section{RNA isolation and quantitative real-time PCR analysis}

Total RNA was isolated from frozen tissue samples using an RNeasy Lipid Tissue mini kit (Qiagen), and then mixed and reacted with a One-Step SYBR ${ }^{\circledR}$ PrimeScript ${ }^{\circledR}$ RT PCR Kit II (Takara Bio, Inc.). Real-time PCR analysis was performed with a LightCycler ${ }^{\circledR} 480$ device (Roche Diagnostics). Relative mRNA transcript levels were calculated using the comparative cycle threshold method, which is based on the difference in cycle threshold values between the expression levels of target mRNA and $18 \mathrm{~S}$ ribosomal RNA, which is used as an internal control. The primers used for real-time PCR analysis are described in Table 1. 
Table 1. Primers used for real-time PCR analysis

\begin{tabular}{|c|c|c|}
\hline Genes & Forward primer & Reverse primer \\
\hline$C C L 2(M C P-1)$ & 5'-AGGTCCCTGTCATGCTTCTGG-3' & 5'-CTGCTGCTGGTGATCCTCTTG-3' \\
\hline CCR2 & 5'-АTTCTCCACACССТGTTTCG-3' & 5'-GATTCCTGGAAGGTGGTCAA-3' \\
\hline IL-6 & 5'-CCACTTCACAAGTCGGAGGCTTA-3' & 5'-GCAAGTGCATCATCGTTGTTCATAC-3' \\
\hline$T N F-\alpha$ & 5'-СССТСАСАСТСАGATCATCTTCT-3' & 5'-GCTACGACGTGGGCTACAG-3' \\
\hline $18 S$ rRNA & 5'-AGGATGTGAAGGATGGGAAG-3' & 5'-ACGAAGGCCCCAAAAGTG-3' \\
\hline
\end{tabular}

CCL2, CC chemokine ligand 2; MCP-1, monocyte chemoattractant protein-1; CCR2, CC chemokine receptor 2; rRNA, ribosomal RNA.

\section{Statistical analysis}

Data are presented as means with their standard errors. Statistical analysis was carried out using the software Prism 5.0 (GraphPad Software, Inc.). The normality of data was assessed using the Kolmogorov-Smirnov test, and all data were normally distributed. Significant differences comparing more than two groups were analysed by the one-way ANOVA, and significant differences between two groups were analysed using the unpaired $t$ test (two-tailed). A $P$ value $<0.05$ was considered to be statistically significant.

\section{Results}

Effect of valine-proline-proline on the physiological parameters of high-fat diet-fed mice

Male C57BL/6J mice (7 weeks of age) were fed with NC (NC group), a HFD and plain tap water (HFD group) or water containing $0.3 \mathrm{mg} \mathrm{VPP} / \mathrm{ml}$ (HFD+VPP group) ad libitum for 10 weeks. Physiological variables of each treatment group are shown in Table 2 . The body weight, epididymal fat weight and liver weight were not significantly different between the HFD and the HFD+VPP groups. There was also no significant difference in the concentrations of plasma total cholesterol, HDL- and LDL-cholesterol, TAG and glucose between the two groups.
Effect of the oral administration of a high-fat diet supplemented with valine-proline-proline on integrin expression in peripheral blood monocytes

Activation of monocytes is closely correlated with systemic inflammatory status. We observed the expression levels of integrins in peripheral blood monocytes after mice were fed a HFD supplemented with or without VPP. As shown in Fig. 1, the HFD induced the relative expression levels of CD11b, CD11c, CD49d, CD18 and CD29. The induction of CD18 was statistically significantly decreased in mice administered with VPP

Effect of the oral administration of a high-fat diet supplemented with valine-proline-proline on the polarisation of monocytes and macrophages in the stromal vascular fractions of epididymal fat

Diet-induced obesity is associated with two phenotypic changes in adipose tissue macrophages: classically activated M1 macrophages and alternatively activated M2 macrophages.

After HFD feeding for 10 weeks, we examined the number of activated monocytes and M1 macrophages, identified as $\mathrm{CD} 11 \mathrm{~b}^{+} / \mathrm{CD} 11 \mathrm{c}^{+}$and $\mathrm{F} 4 / 80^{+} / \mathrm{CD} 11 \mathrm{c}^{+}$, respectively, in the SVF of epididymal fat tissue in the NC, HFD and HFD+VPP groups. The number of activated monocytes

Table 2. Body weight (BW), tissue weight and plasma parameters of normal chow (NC)-fed mice, high-fat diet (HFD)-fed mice and HFD+valine-proline-proline (VPP)-fed mice for a test period of 10 weeks

(Mean values with their standard errors, $n 7$ )

\begin{tabular}{|c|c|c|c|c|c|c|}
\hline & \multicolumn{2}{|c|}{ NC } & \multicolumn{2}{|c|}{ HFD } & \multicolumn{2}{|c|}{ HFD+VPP } \\
\hline & Mean & SEM & Mean & SEM & Mean & SEM \\
\hline Final BW (g) & 31.8 & 0.7 & 35.5 & 0.9 & $36 \cdot 6$ & 0.6 \\
\hline BW gain (g) & $8 \cdot 1$ & 0.5 & $11 \cdot 7$ & 0.9 & $12 \cdot 6$ & 0.6 \\
\hline Epididymal fat weight (g) & 0.44 & 0.03 & 1.5 & 0.2 & 1.3 & 0.2 \\
\hline Epididymal fat weight/BW (\%) & 0.014 & 0.001 & 0.040 & 0.004 & 0.036 & 0.005 \\
\hline Liver weight $(\mathrm{g})$ & 1.7 & 0.1 & 1.9 & 0.1 & $2 \cdot 0$ & 0.1 \\
\hline Liver weight/BW (\%) & 0.054 & 0.002 & 0.052 & 0.002 & 0.055 & 0.002 \\
\hline Plasma glucose $(\mathrm{mmol} / \mathrm{l})$ & 9.47 & 0.45 & $10 \cdot 0$ & 0.9 & $12 \cdot 1$ & 0.5 \\
\hline Total cholesterol (mmol/l) & 1.28 & 0.07 & 2.77 & 0.22 & $2 \cdot 88$ & 0.14 \\
\hline TAG (mmol/l) & 1.08 & 0.08 & 0.72 & $0 \cdot 11$ & 0.67 & 0.07 \\
\hline LDL-cholesterol (mmol/l) & 0.11 & 0.01 & 0.29 & 0.02 & 0.32 & 0.02 \\
\hline HDL-cholesterol (mmol/l) & 0.68 & 0.03 & 1.42 & 0.15 & 1.52 & 0.08 \\
\hline NEFA (mmol/l) & 119 & 20 & 157 & 19 & 123 & 14 \\
\hline
\end{tabular}




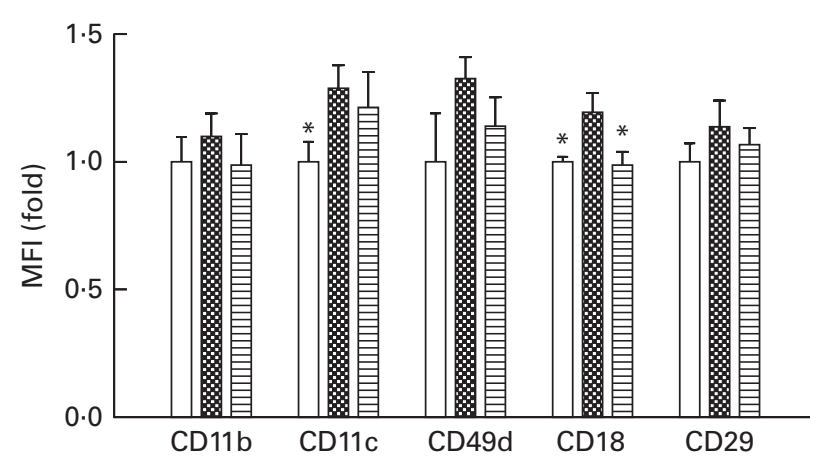

Fig. 1. Dietary fat and valine-proline-proline (VPP) modulates the expression levels of specific integrins on the surface of mouse peripheral blood monocytes. Flow cytometric analysis was used to assess the abundance of individual integrin species on the cell surface of monocytes from the peripheral blood of normal chow (NC)-fed mice ( $\square$ ), high-fat diet (HFD)-fed mice (Q) and HFD+VPP-fed mice (目). Each group was treated for a test period of 10 weeks ( $n$ 5-8 animals per group), and specific integrins were identified using cognate monoclonal antibodies as described in the Materials and methods section. Values are means, with their standard errors represented by vertical bars. * Mean value was significantly different from that of the HFD group $(P<0.05)$. MFI, mean fluorescence intensity.

$\left(\mathrm{CD} 11 \mathrm{~b}^{+} / \mathrm{CD} 11 \mathrm{c}^{+}\right.$cells) was significantly increased in response to the HFD in the SVF of epididymal fat tissue in the HFD group, when compared with the control group. Treatment of HFD-fed mice with VPP did not significantly reduce the number of activated monocytes. The number of M1 macrophages $\left(\mathrm{F} 4 / 80^{+} / \mathrm{CD} 11 \mathrm{c}^{+}\right.$cells) in the HFD group was significantly increased when compared with the control group. However, this increase was significantly reduced by the administration of VPP. By contrast, the number of M2 macrophages (F4/80 $/$ CD204 ${ }^{+}$cells) did not differ among the three groups (Fig. 2).

\section{Valine-proline-proline reduces macrophage infiltration into the adipose tissue of high-fat diet-fed mice}

To directly observe the inflammation in adipose tissue, we counted the number of crown-like structures in the epididymal fat tissue of HFD-fed mice. The crown-like structures comprised $\mathrm{F} 4 / 80^{+}$adipose tissue macrophages surrounding an adipocyte ${ }^{(25,26)}$, which are rarely observed in those of standard diet-fed mice ${ }^{(1)}$. The number of crown-like structures in the HFD+VPP group was significantly decreased compared with that in the HFD group (Fig. 3(a)), which was consistent with the reduction in the number of pro-inflammatory M1 macrophages (Fig. 2).

\section{Valine-proline-proline suppresses inflammatory gene expression in adipose tissue}

M1 macrophages in adipose tissue secrete pro-inflammatory cytokines, such as TNF- $\alpha$, IL- 6 and monocyte chemoattractant protein-1 (MCP-1), thus contributing to the induction of insulin resistance ${ }^{(24,27,28)}$. We then measured the expression levels of mRNA prepared from the adipose tissue of mice fed the HFD supplemented with or without VPP.
The expression of $M C P-1$, a monocyte or macrophage chemotactic factor, was significantly reduced in the HFD+VPP group when compared with the HFD group (Fig. 4). This result suggests a causative role of $\mathrm{MCP}-1$ in the recruitment of M1 macrophages in adipose tissue. These VPP-mediated reductions in the expression of $M C P-1$ play a key role in the stabilisation of adipose tissue inflammation via M1 macrophages.

\section{Discussion}

In the present study, oral administration of a naturally occurring tripeptide, VPP, diminished obesity-related chronic inflammation induced by HFD feeding in mice. We observed a local anti-inflammatory effect of VPP on adipose tissue via the inhibition of the accumulation of pro-inflammatory macrophages in the SVF, without any change in body weight and epididymal fat weight. Anti-inflammatory properties of food-derived peptides have been reported in several previous studies ${ }^{(12,13,29-31)}$. However, to our knowledge, the present study is the first aimed at examining the antiinflammatory effect exerted by the oral administration of such peptides on adipose tissue inflammation and proinflammatory macrophages.

The HFD enhances the expression levels of integrins in monocytes and increases the amount of MCP-1 in the peripheral blood. In addition, it leads to the accumulation of activated monocytes and M1 macrophages in visceral adipose tissue $^{(16,18,32)}$. In the present study, we examined the number of M1 macrophages that infiltrated into epididymal fat tissue, as a function of diet and casein-derived peptides. An important question is whether the increase that we observed, in the levels of M1 macrophages infiltrating into adipose tissue in HFD-fed mice, is due to a phenotypic change from M2 to M1 macrophages, or whether circulating pro-inflammatory monocytes are recruited into adipose tissue directly from the bloodstream ${ }^{(33,34)}$. In the present study, we found that HFD feeding resulted in an increase in M1 macrophages $\left(\mathrm{F} 4 / 80^{+} / \mathrm{CD}_{11 \mathrm{c}^{+}}\right.$cells) in adipose tissue, as reported

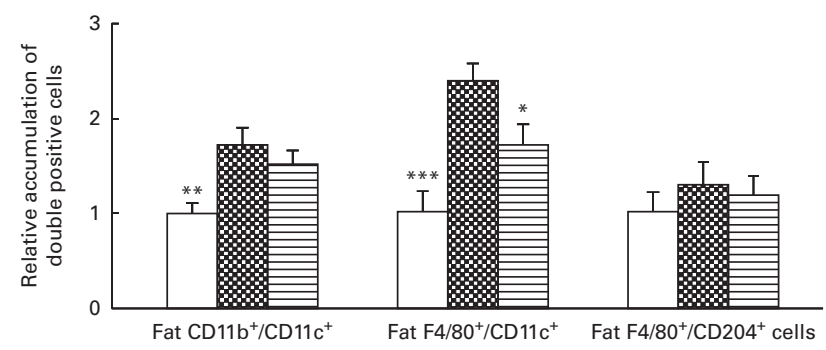

Fig. 2. Valine-proline-proline (VPP) attenuates high-fat diet (HFD)-induced macrophage infiltration into adipose tissue. After a test period of 10 weeks, stromal vascular fractions were isolated from the epididymal visceral adipose tissue of the different treatment groups (normal chow (NC)-fed mice ( $\square$ ), high-fat diet (HFD)-fed mice ( $\mathrm{O}$ ) and HFD+VPP-fed mice (目)), and the relative amounts of activated monocytes $\left(\mathrm{CD} 11 \mathrm{~b}^{+} / \mathrm{CD} 11 \mathrm{c}^{+}\right.$cells), M1 macrophages ( $\mathrm{F} 4 / 80^{+} / \mathrm{CD} 11 \mathrm{c}^{+}$cells) and $\mathrm{M} 2$ macrophages $\left(\mathrm{F} 4 / 80^{+} / \mathrm{CD} 204^{+}\right.$cells) were determined by flow cytometric analysis. Values are means ( $n$ 6-7 animals per group), with their standard errors represented by vertical bars. Mean value was significantly different from that of the HFD group: ${ }^{*} P<0.05$, ${ }^{\star \star} P<0.01,{ }^{\star \star \star} P<0.001$. 
(a)

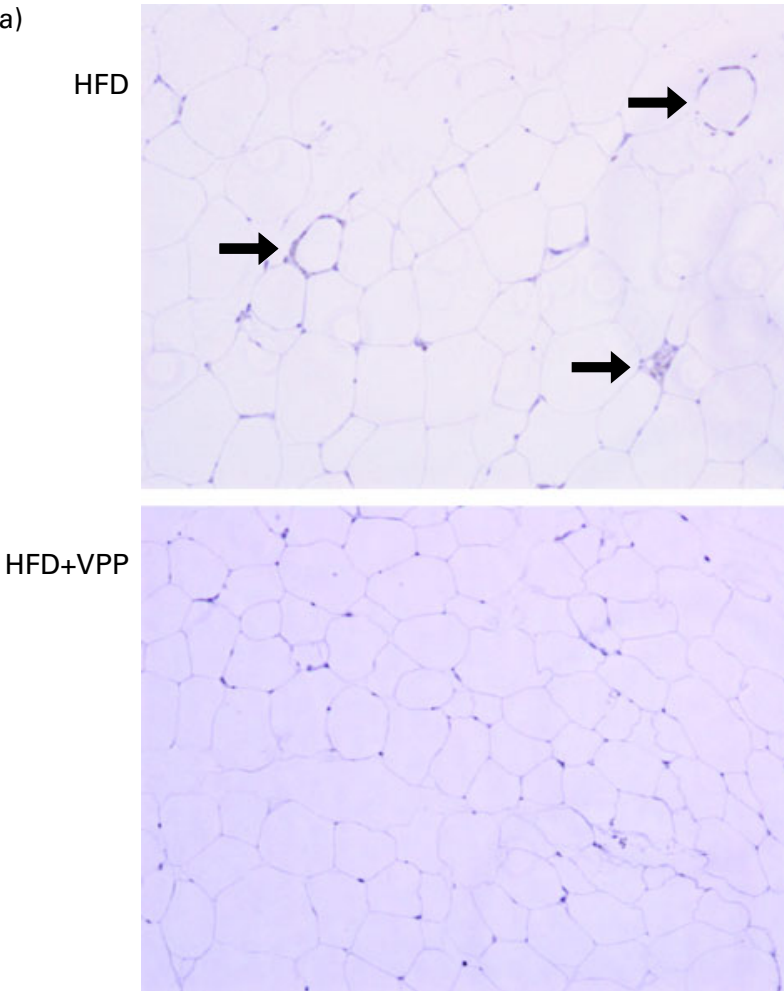

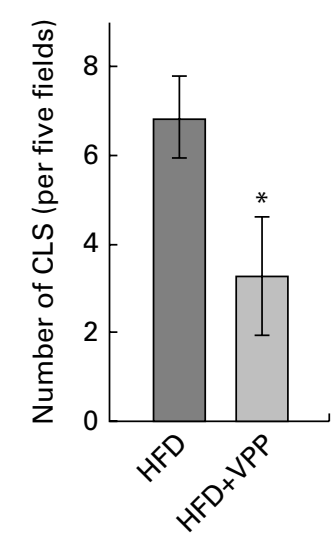

Fig. 3. Valine-proline-proline (VPP) reduces adipose tissue inflammation and macrophage accumulation in the adipose tissue of high-fat diet (HFD)-fed mice. The formation of crown-like structures (CLS) in the epididymal adipose tissue of HFD-fed and HFD+VPP-fed mice for a test period of 10 weeks was analysed. (a) Histology of the epididymal adipose tissue of HFD-fed and HFD+VPP-fed mice. Epididymal fat tissue was stained with the anti-F4/80 antibody. The arrows indicate the CLS. (b) Quantification of the number of CLS in HFD-fed and HFD+VPP-fed mice. The number of CLS was counted as described in the Materials and methods section. Values are means ( $n 7$ animals per group), with their standard errors represented by vertical bars.

* Mean value was significantly different from that of the HFD group $(P<0.05)$.

A colour version of this figure can be found online at http://www.journals. cambridge.org/bjn

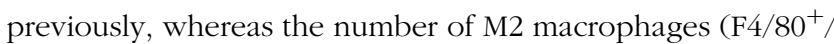
CD $204^{+}$cells) was not affected, even in the HFD group. This strongly suggests that the net increase in the infiltration of M1 macrophages may be due to the direct recruitment of circulating pro-inflammatory monocytes or macrophages into adipose tissue. The administration of VPP significantly decreased the number of M1 macrophages but not M2 macrophages in epididymal fat tissue, in response to the HFD treatment. This indicates a role of VPP in regulating the recruitment of pro-inflammatory cells and, therefore, in the regulation of diet-induced inflammatory changes in adipose tissue.

Previous studies have indicated that the MCP-1/CC chemokine receptor 2 (CCR2) pathway contributes to macrophage polarisation in adipose tissue ${ }^{(35)}$. In agreement with that study, we found that the expression levels of $M C P-1, C C R 2$ and $I L-6$ in adipose tissue were increased in response to the HFD treatment, and that VPP reduced the HFD-dependent expression levels of $M C P-1$. These data suggest that VPP modulates HFD-induced MCP-1/CCR2 signalling in adipose tissue. However, the effect of VPP on the reduction in M1 macrophage infiltration was relatively small when compared with its effect on the expression levels of $M C P-1$. In the present study, we failed to observe a systemic effect of VPP on the amount of MCP-1 in the plasma of HFD-fed mice (data not shown). Therefore, another mechanism independent of MCP-1 may also contribute to the activation of M1 macrophages $^{(36,37)}$.

Monocyte-endothelial interactions, including tethering, rolling, firm adhesion and extravasation, form one of the crucial steps leading to atherosclerosis, which is mediated by various adhesion molecules expressed on leucocytes and endothelial cells. In the present study, we investigated whether the expression levels of integrins in monocytes were altered in mice fed the HFD supplemented with or without VPP. We found that the expression level of CD18 was significantly decreased by the administration of VPP (Fig. 1). It has previously been reported that mRNA levels of intercellular adhesion molecule-1 (ICAM-1) were increased specifically in the visceral adipose tissue of male wild-type mice after 3 weeks of HFD feeding ${ }^{(17)}$. In addition, increased leucocyte-endothelial cell interaction was observed in the microcirculation of visceral adipose tissue in $o b / o b$ and

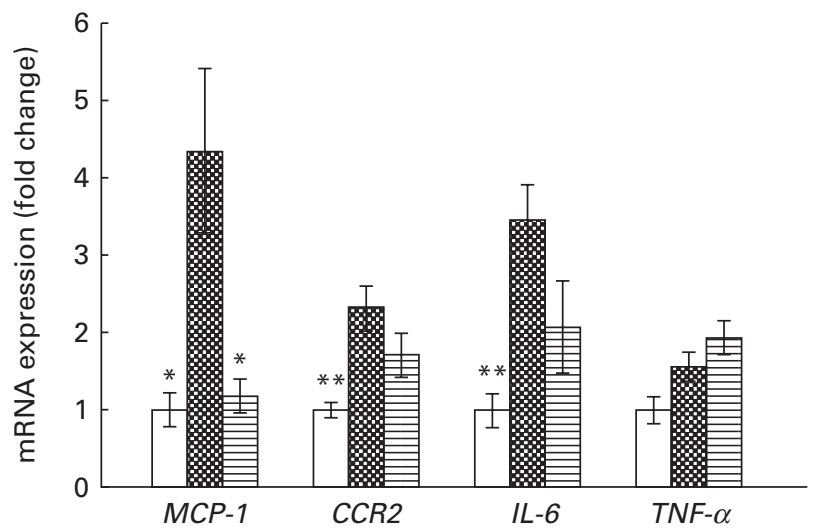

Fig. 4. Valine-proline-proline (VPP) suppresses the expression of pro-inflammatory cytokines and the chemokine receptor in adipose tissue. mRNA expression of inflammatory markers in epididymal adipose tissue from mice fed with normal chow (NC; $\square$ ), a high-fat diet (HFD; 图) and a HFD+VPP (目) for a test period of 10 weeks. Values are means ( $n 5$ animals per group), with their standard errors represented by vertical bars. Mean value was significantly different from that of the HFD group: ${ }^{*} P<0.05$, ${ }^{* *} P<0.01$. MCP-1, monocyte chemoattractant protein-1; CCR2, CC chemokine receptor 2 . 
HFD-induced obese mice, as well as up-regulated expression of ICAM-1, E-selectin and P-selectin on endothelial cells $\left(\mathrm{CD} 31^{+}\right.$cells) of visceral adipose tissue in $o b / o b$ mice ${ }^{(38)}$. The $\beta 2$-integrin, also known as CD18, forms a dimer with the $\alpha$-integrins CD11a, CD11b and CD11c, and the heterodimer binds to the cognate ligands ICAM-1 (CD11a and CD11b) and vascular cell adhesion molecule-1 (CD11c) on the endothelial surface, thus contributing to the adhesion between monocytes and endothelial cells. As we have previously found that IPP and VPP significantly reduced PMAstimulated THP-1 cell adhesion to activated human umbilical vein endothelial cells via $\beta 2$-integrin in THP- 1 cells ${ }^{(13)}$, the present observations generalise these findings to the microvasculature of adipose tissue in a whole animal model.

Previous studies have shown that VPP was absorbed intact through the intestine, and moved into the systemic circulation $^{(39-42)}$. After entering the plasma, these peptides become accessible to adipose tissue. The previous reports have also shown the successful detection of IPP and VPP in the aorta of spontaneously hypertensive rats after the oral administration of these peptides ${ }^{(39,42)}$, and cyanine 3 (Cy3)-labelled derivatives of both peptides were localised in the endothelial cells of arterial vessels in rats ${ }^{(42)}$. Therefore, VPP may accumulate in the microvascular vessels of adipose tissue, and exert an in situ anti-inflammatory effect.

In conclusion, the results of the present study highlight the possibility that administration of VPP can ameliorate chronic inflammation induced by a HFD, at least in adipose tissue. The underlying mechanism by which VPP supplementation leads to the decrease in the accumulation of pro-inflammatory macrophages in the SVF involves the inhibition of the expression levels of pro-inflammatory cytokines and chemokines. Further studies are needed to elucidate the precise molecular mechanisms by which VPP negatively regulates inflammation. Ultimately, such understanding may eventually lead to the development of a preventive strategy for chronic inflammation-related diseases.

\section{Acknowledgements}

The present study was supported in part by Calpis Company Limited, Tokyo, Japan.

The authors' contributions are as follows: K. A. and M. Y. designed the research; K. A. and M. O. conducted the research and analysed the data; K. A. and M. Y. wrote the manuscript; K. A., M. O. and M. Y. reviewed and edited the manuscript. All authors read and approved the final manuscript.

K. A. is affiliated with Calpis Company Limited. The rest of the authors declare that there are no conflicts of interest.

\section{References}

1. Weisberg SP, McCann D, Desai M, et al. (2003) Obesity is associated with macrophage accumulation in adipose tissue. J Clin Invest 112, 1796-1808.

2. Van Gaal LF, Mertens IL \& De Block CE (2006) Mechanisms linking obesity with cardiovascular disease. Nature $\mathbf{4 4 4}$ $875-880$
3. Guilherme A, Virbasius JV, Puri V, et al. (2008) Adipocyte dysfunctions linking obesity to insulin resistance and type 2 diabetes. Nat Rev Mol Cell Biol 9, 367-377.

4. Nakamura Y, Yamamoto N, Sakai K, et al. (1995) Purification and characterization of angiotensin I-converting enzyme inhibitors from sour milk. J Dairy Sci 78, 777-783.

5. Mizuno S, Nishimura S, Matsuura K, et al. (2004) Release of short and proline-rich antihypertensive peptides from casein hydrolysate with an Aspergillus oryzae protease. J Dairy Sci 87, 3183-3188.

6. Cicero AF, Gerocarni B, Laghi L, et al. (2011) Blood pressure lowering effect of lactotripeptides assumed as functional foods: a meta-analysis of current available clinical trials. J Hum Hypertens 25, 425-436.

7. Cicero AF, Aubin F, Azais-Braesco V, et al. (2013) Do the lactotripeptides isoleucine-proline-proline and valine-prolineproline reduce systolic blood pressure in European subjects? A meta-analysis of randomized controlled trials. Am J Hypertens 26, 442-449.

8. Hirota T, Nonaka A, Matsushita A, et al. (2011) Milk caseinderived tripeptides, VPP and IPP induced NO production in cultured endothelial cells and endothelium-dependent relaxation of isolated aortic rings. Heart Vessels 26, 549-556.

9. Hirota T, Ohki K, Kawagishi R, et al. (2007) Casein hydrolysate containing the antihypertensive tripeptides Val-Pro-Pro and Ile-Pro-Pro improves vascular endothelial function independent of blood pressure-lowering effects: contribution of the inhibitory action of angiotensin-converting enzyme. Hypertens Res 30, 489-496.

10. Yoshizawa M, Maeda S, Miyaki A, et al. (2009) Additive beneficial effects of lactotripeptides and aerobic exercise on arterial compliance in postmenopausal women. Am J Physiol Heart Circ Physiol 297, H1899-H1903.

11. Nakamura T, Mizutani J, Ohki K, et al. (2011) Casein hydrolysate containing Val-Pro-Pro and Ile-Pro-Pro improves central blood pressure and arterial stiffness in hypertensive subjects: a randomized, double-blind, placebo-controlled trial. Atherosclerosis 219, 298-303.

12. Nakamura T, Hirota T, Mizushima K, et al. (2013) Milkderived peptides, Val-Pro-Pro and Ile-Pro-Pro, attenuate atherosclerosis development in apolipoprotein E-deficient mice: a preliminary study. J Med Food 16, 396-403.

13. Aihara K, Ishii H \& Yoshida M (2009) Casein-derived tripeptide, Val-Pro-Pro (VPP), modulates monocyte adhesion to vascular endothelium. $J$ Atheroscler Thromb 16, 594-603.

14. Webster GA, Bowles MJ, Karim MS, et al. (1995) Flow cytometric analysis of peripheral blood lymphocyte subset light scatter characteristics as a means of monitoring the development of rat small bowel allograft rejection. Clin Exp Immunol 100, 536-542.

15. Lazarus AH, Ellis J, Blanchette V, et al. (1998) Permeabilization and fixation conditions for intracellular flow cytometric detection of the T-cell receptor zeta chain and other intracellular proteins in lymphocyte subpopulations. Cytometry 32, 206-213.

16. Hagita S, Osaka M, Shimokado K, et al. (2011) Adipose inflammation initiates recruitment of leukocytes to mouse femoral artery: role of adipo-vascular axis in chronic inflammation. PLOS ONE 6, e19871.

17. Brake DK, Smith EO, Mersmann H, et al. (2006) ICAM-1 expression in adipose tissue: effects of diet-induced obesity in mice. Am J Physiol Cell Physiol 291, C1232-C1239.

18. Wu H, Perrard XD, Wang Q, et al. (2010) CD11c expression in adipose tissue and blood and its role in diet-induced obesity. Arterioscler Thromb Vasc Biol 30, 186-192. 
19. Ito A, Suganami T, Yamauchi A, et al. (2008) Role of CC chemokine receptor 2 in bone marrow cells in the recruitment of macrophages into obese adipose tissue. $J$ Biol Chem 283, 35715-35723.

20. Kurahara H, Shinchi H, Mataki Y, et al. (2011) Significance of M2-polarized tumor-associated macrophage in pancreatic cancer. J Surg Res 167, e211-e219.

21. Komohara Y, Ohnishi K, Kuratsu J, et al. (2008) Possible involvement of the M2 anti-inflammatory macrophage phenotype in growth of human gliomas. J Pathol 216, 15-24.

22. Patsouris D, Neels JG, Fan W, et al. (2009) Glucocorticoids and thiazolidinediones interfere with adipocyte-mediated macrophage chemotaxis and recruitment. J Biol Chem 284, 31223-31235.

23. Nguyen MT, Favelyukis S, Nguyen AK, et al. (2007) A subpopulation of macrophages infiltrates hypertrophic adipose tissue and is activated by free fatty acids via Toll-like receptors 2 and 4 and JNK-dependent pathways. J Biol Chem 282, 35279-35292

24. Lumeng CN, Bodzin JL \& Saltiel AR (2007) Obesity induces a phenotypic switch in adipose tissue macrophage polarization. J Clin Invest 117, 175-184.

25. Cinti S, Mitchell G, Barbatelli G, et al. (2005) Adipocyte death defines macrophage localization and function in adipose tissue of obese mice and humans. J Lipid Res 46, 2347-2355.

26. Nishimura S, Manabe I, Nagasaki M, et al. (2007) Adipogenesis in obesity requires close interplay between differentiating adipocytes, stromal cells, and blood vessels. Diabetes 56, 1517-1526.

27. Gordon S \& Taylor PR (2005) Monocyte and macrophage heterogeneity. Nat Rev Immunol 5, 953-964.

28. Mantovani A, Sica A, Sozzani S, et al. (2004) The chemokine system in diverse forms of macrophage activation and polarization. Trends Immunol 25, 677-686.

29. Hernández-Ledesma B, Hsieh CC \& de Lumen BO (2013) Chemopreventive properties of Peptide Lunasin: a review. Protein Pept Lett 20, 424-432.

30. Kovacs-Nolan J, Zhang H, Ibuki M, et al. (2012) The PepT1-transportable soy tripeptide VPY reduces intestinal inflammation. Biochim Biophys Acta 1820, 1753-1763.

31. Cam A \& de Mejia EG (2012) Role of dietary proteins and peptides in cardiovascular disease. Mol Nutr Food Res 56, 53-66.
32. Wu H, Gower RM, Wang $\mathrm{H}$, et al. (2009) Functional role of $\mathrm{CD}_{11 \mathrm{c}^{+}}$monocytes in atherogenesis associated with hypercholesterolemia. Circulation 119, 2708-2717.

33. Stout RD, Jiang C, Matta B, et al. (2005) Macrophages sequentially change their functional phenotype in response to changes in microenvironmental influences. I Immunol 175, 342-349.

34. Lumeng CN, DelProposto JB, Westcott DJ, et al. (2008) Phenotypic switching of adipose tissue macrophages with obesity is generated by spatiotemporal differences in macrophage subtypes. Diabetes 57, 3239-3246.

35. Weisberg SP, Hunter D, Huber R, et al. (2006) CCR2 modulates inflammatory and metabolic effects of high-fat feeding. I Clin Invest 116, 115-124.

36. Inouye KE, Shi H, Howard JK, et al. (2007) Absence of CC chemokine ligand 2 does not limit obesity-associated infiltration of macrophages into adipose tissue. Diabetes 56, 2242-2250.

37. Kirk EA, Sagawa ZK, McDonald TO, et al. (2008) Monocyte chemoattractant protein deficiency fails to restrain macrophage infiltration into adipose tissue [corrected]. Diabetes 57, 1254-1261.

38. Nishimura S, Manabe I, Nagasaki M, et al. (2008) In vivo imaging in mice reveals local cell dynamics and inflammation in obese adipose tissue. J Clin Invest 118, $710-721$

39. Masuda O, Nakamura Y \& Takano T (1996) Antihypertensive peptides are present in aorta after oral administration of sour milk containing these peptides to spontaneously hypertensive rats. J Nutr 126, 3063-3068.

40. Satake M, Enjoh M, Nakamura Y, et al. (2002) Transepithelial transport of the bioactive tripeptide, Val-Pro-Pro, in human intestinal Caco-2 cell monolayers. Biosci Biotechnol Biochem 66, 378-384.

41. Ohsawa K, Satsu H, Ohki K, et al. (2008) Producibility and digestibility of antihypertensive beta-casein tripeptides, Val-Pro-Pro and Ile-Pro-Pro, in the gastrointestinal tract: analyses using an in vitro model of mammalian gastrointestinal digestion. J Agric Food Chem 56, 854-858.

42. Kawaguchi K, Nakamura T, Kamiie J, et al. (2012) Accumulation of ACE inhibitory tripeptides, Val-Pro-Pro and Ile-Pro-Pro, in vascular endothelial cells. Biosci Biotechnol Biochem 76, 1792-1795. 